\title{
A Comparative Study of Hierarchical Clustering based Routing Protocols in WSN: A Survey
}

\author{
Supriya Bhagat \\ M.Tech Scholar \\ Department of Computer Science \& Engineering \\ Guru Nanak Dev University, Amritsar
}

\author{
Kamaljit Kaur \\ Assistant Professor \\ Department of Computer Science \& Engineering, \\ Guru Nanak Dev University, \\ Amritsar
}

\begin{abstract}
Development of wireless sensor network technologies have presented a availability of tiny sensor nodes along with the ability regarding sensing various physical as well as atmospheric circumstances, data handling and wireless communication. A number of detecting abilities affect the abundance application zones. On the other hand, a function associated wireless sensor networks demand additional efficient techniques for data sending as well as dispensation. In WSN, the sensor nodes include restricted transmission range; dispensation, storage abilities and energy resources also are restricted. Therefore various routing protocols are designed for the sensor network to overcome these problems. Among the routing protocols one is hierarchical routing which follows a clustering mechanism and also considered to be more resourceful in regards to energy as well as scalability. In this paper, we are surveyed energy efficient hierarchical cluster-based routing protocol that periodically selects cluster head as per the hybridization of their residual energy.
\end{abstract}

\section{Keywords}

WSN, Routing protocols, Energy efficiency, clustering

\section{INTRODUCTION}

A Wireless Sensor Network is a self-arranging network which composes the tiny sensor nodes communicating through broadcasting signal, embedded computing and connectivity. The wireless sensors network comprise sensor nodes arranged on the geographical area for observing either physical or environment situations like sound, pressure, motion, pollutant. Collecting and forwarding information is done through the base station. The capability of sensor node is to sense as well as process the data.

An average sensor node comprises of four constructing blocks: power unit, communication unit, processing unit and sensing unit [41] which are presented in fig1. Sensors and $\mathrm{ADC}$ are the two subunits of sensing unit. In node the sensing component processes a number of physical attributes including temperature. The collecting and handling captured data from its surrounding is responsible in processing component. The sensor node is communicating with each other in order to aggregating the data. The main work of sensor node is usually sended the collected data among cluster head to base station or one node to another node through the wireless communication cost. The data is then sending to the internet with the help of gateway as user is straight linked to a internet. These three component sensor node, processing as well as communication requires power to utility as estimated, and the forth one power component is surely responsible for providing the energy to the three other component. The power mode might be held up by power scavenging unit just like solar. Location finding system and mobilizer both are the other subunit of sensor node where location finding system must be require to provide the knowledge of location for the sensor routing techniques as well as for sensing tasks and Mobilizer is required to carry out the movement of sensor nodes. WSN generates a local network hierarchy on multiple levels signified by nodes elected by definite conditions that are collecting as well as transmitting information towards central base station (BS). [41]

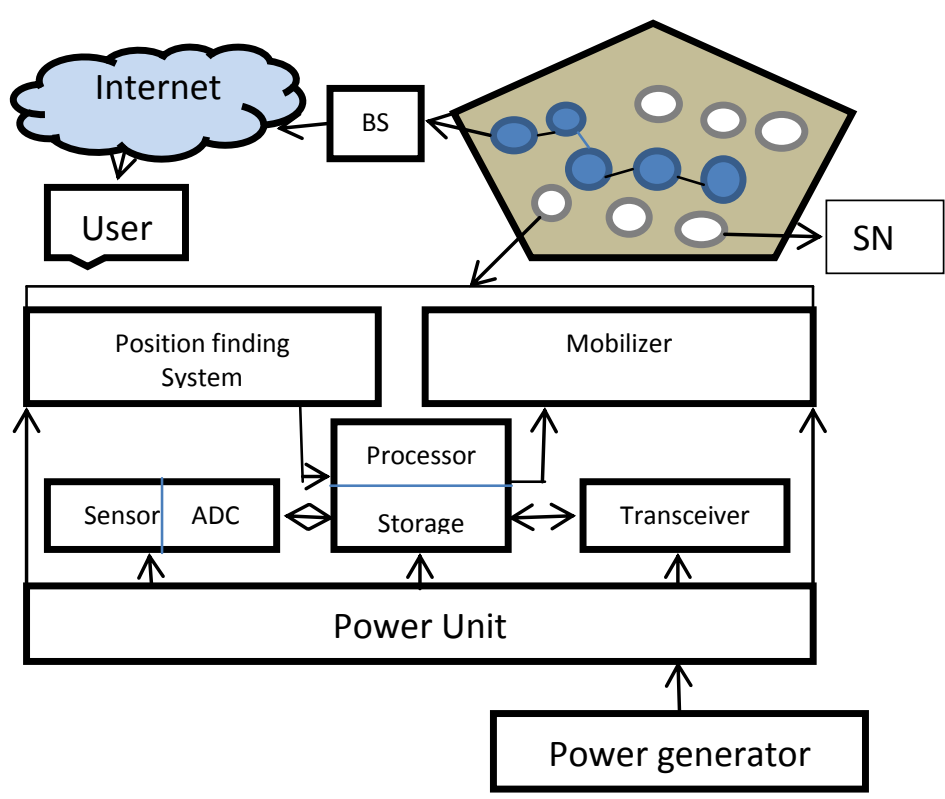

Fig.1 Components of sensor node

\subsection{Applications of WSN}

Nowadays a Wireless Sensor Networks have discovered their own way into a multiple of applications as well as systems along with a hugely varying requirements and characteristics. The applications of wireless sensor network mainly include health, military environment, home and other commercial areas.

\subsubsection{Area Monitoring}

In this type of application, the sensor network can be integrated over an area where certain spectacle has to be observed. This type of network is uses to command control and communicate.

\subsubsection{Health care monitoring}

This type of application is used to tracking and monitors doctors or patients inside the hospital. Wearable and implanted are the main devices of medical applications 
Wearable devices are utilized on the human frame and implanted medicinal devices are implanted inside human body.

\subsubsection{Environment/Earth monitoring}

These types of applications are used for monitoring the environment parameters. Example - landslide detection which is used to sense the slight movement of mud and variations in numerous parameters. These types of application are also including the sensing of glaciers, volcanoes, earthquakes etc. Some major monitoring includes air pollution and greenhouse.

\subsubsection{Waste water monitoring}

This type of application would include of monitoring the quality and level of water like testing the quality of surface water.

\subsubsection{Structure health monitoring}

Wireless sensor networks can be used to examine the circumstance of civil structure as well as associated geophysical processes near to real time through data sorting, using suitably interfaced sensors.

\section{ROUTING PROTOCOLS}

Routing is the process of choosing a route within a network along which to transmit network traffic. The main working of sensor network routing protocol is to sended data through sensor nodes toward the sink node in an energy awareness method which maximizes the network lifetime of the different sensors. There are different varieties of routing protocols which can be categorized as data centric, hierarchical, location and query based protocol dependent upon network structure and protocol operations as presented in fig2.

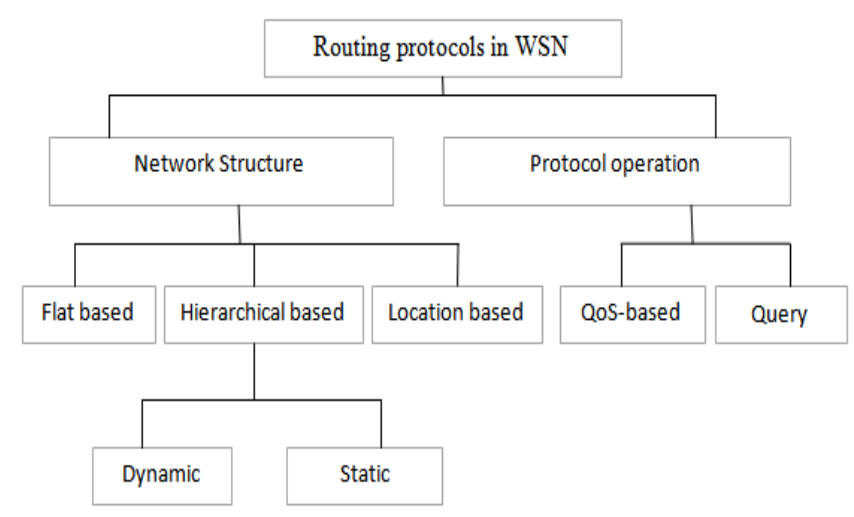

Fig2: Classification of routing protocols

In Flat routing protocols, the sensor nodes are positioned consistently i.e., everyone node reaches at the same stage into a network. In Location based protocols, sensor nodes are tend to be resolved by using its location[38]. The information related to location with regard to sensor nodes can be very important to sensor networks by way of the majority of routing protocols in order to estimate the distance among two particularly nodes to make sure that energy depletion must be calculated. In the query based routing, while sending data, the network balances among energy consumption as well as quality of service matrix such as delay, energy. Hierarchical Protocols are more energy efficient protocols compared to other protocols. [34] [35]. In this paper we are giving survey of hierarchical protocols only that is why we have specify importance and significance of such protocols here.

\subsection{Hierarchical routing protocols}

Standard routing protocols might not be best in energy depletion. Most protocols are not as energy efficient compared to Hierarchical routing protocols. Hierarchical routing follows the clustering mechanism which is considered to be resourceful with regards to power plus scalability. Use of such method minimizes the depletion of energy increasingly in collecting and distributing data. Here the nodes tend to be organized into clusters as well as nodes which may have highest energy are called Cluster head. Cluster Head is mainly liable for gathering data from nodes through cluster and eliminating idleness among collected data to diminish energy constraint for sending of data packets through cluster head to base station [24].

Many hierarchical routing protocols are discovered to overcome the problem of energy depletion which is described below.

\subsubsection{Leach}

Leach is usually one of the essential hierarchical protocols which can be used to minimize the energy utilization in a network and this type of protocol can only provision the single hop communication. In order to reduce power consumption in a network, In Leach protocol all nodes are supposed to be static and energy constraint and nodes send data directly towards base station. Set up and steady are the two main phases of Leach protocol. The cluster head is elected in setup phase and in case of steady phase, the cluster head is retained where information is communicated among nodes. The trade of cluster head is to gathering plus sending the data towards base station [1].

\subsubsection{Pegasis}

Pegasis is usually a chain type protocol which is advancement over leach. Unlike Leach, it ignores cluster formation. In this protocol, only one node is chosen a head node which sends the merged data to the sink for every round. In Pegasis every node communicates through their near neighbor and then takes turn while transmitting to the base station. It works well as compare to LEACH. Pegasis uses a greedy approach and in case if there is any node failure then it bypasses that node. In Pegasis the Data fusion includes a number of data packages from dissimilar sensor dimensions to create a distinct packet. The basic idea of this protocol is to create a chain between the sensor nodes so that everyone node collecting as well as forwarding the data to their near neighbor [6].

\subsubsection{Teen (Threshold Sensitive Energy Efficient Sensor Network Protocol)}

Teen protocol is a clustering communication protocol which can be basically designed for the reactive type networks. This protocol is usually for a basic temperature detecting applications. These type of protocols among these networks should be calculated in a manner that the minimal power of every sensor nodes can be resourcefully used. TEEN protocol can be well suited for time crucial applications. The operation associated with TEEN is ideal for two modes- hard mode and soft mode. The hard mode must be focused among middling value of the lowermost as well as the maximum possible temperature, $100^{\circ} \mathrm{F}$. The soft mode must be focused at $2^{\circ} \mathrm{F}$. The modification can usually made in the value of hard as well as soft mode values so that we can resistor the number of packet communications [14] 


\subsubsection{APTEEN}

APTEEN is usually an advancement of a TEEN protocol. APTEEN is a fusion routing protocol which permits for widespread information retrieved. In such kind of networks the nodes not even respond on time based applications but also offer a complete image of the network to the constant intervals. The node can transmits in time slot only if both values larger than a hard threshold or value differs from last transmitted value (SV) by more than a soft. APTTEN is designed with a elasticity of permitting the user to create the time interval as well as mode values for different types of characteristics. The Power consumption of APTEEN is better than LEACH but not as good as TEEN [14] [15].

\subsubsection{Hybrid Energy Efficient Distributed Protocol} HEED is usually for the ad hoc sensor networks. In HEED all nodes are supposed to be homogeneous i.e. all sensor nodes have the same initial energy. HEED prolongs the basic arrangement regarding Leach by utilizing remaining power as well as degree of node or solidity like a metric regarding cluster election to attain power assessment. In HEED, all nodes are chooses themselves as a cluster head according to their remaining power as well as the node degree for cluster election to attain power assessment. HEED is a multi-hop cluster-based protocol. The HEED has two main parametersfirst parameter is dependent upon remaining power of all sensor nodes as well as second is the intra-cluster communiqué cost as an occupation of the cluster solidity. The HEED clustering increases lifespan of sensor network over Leach because Leach casually elects $\mathrm{CHs}$ which can affect the earlier demise of some nodes [19].

\subsubsection{Distance Base Cluster Head Protocol}

In DBCH the nodes may elect a cluster head according to the distance between its $\mathrm{CH}$ towards sink (base station) and distance between node itself as well as sink .Its aim is to protect the total energy cost of each and every sensor networks and also extend the lifespan of sensor network. In this, the nodes elect the cluster head which lies nearby to the midpoint towards base station and sensor nodes, which directly shows the increased network survivability [12].

\subsubsection{Quadrature Leach}

Quadrature Leach increases stability period, network lifetime and throughput moderately considerable. In this, they partition the network into four quadrants to acquire better clustering. TDMA approach is used in Quadratic Leach. Fewer error chances, Fast processing and less time consumption are the advantages of Q-Leach [7].

\subsubsection{I-Leach}

I-Leach is a new and different type of the self-organized routing algorithm which strongly uses the power and also owns benefits of tree as well as hierarchical routing. Improved Leach elect the cluster head whose node have the maximum residual power and also has more neighbors and lower distance from the base station. Improved Leach also succeeds the clusters and sensor nodes to minimize the energy depletion of wireless sensor network. In I-Leach each and every node has the same preliminary energy and fixed. Every sensor node has a distinctive identification and known its current location as well as its remaining energy [3].

\subsubsection{C-Leach (Centralized Leach)}

Leach-C is better for small networks. Leach-C works in two rings first ring is the cluster Setup phase and second ring must be Steady-State phase. Setup phase includes cluster head announcement as well as arrangement of different nodes within each and every cluster and Steady-State phase contains the communication of the data toward nodes to cluster head as well as cluster head towards base station. The Base station organized the dynamic clustering protocol (BCDCP) which can be dependent on the base station to elect the cluster head from a number of sensor nodes [10].

\subsubsection{Leach-F}

Leach-F is similar as Leach-C protocols. In Leach-C the cluster head election must be concerned but in case of Leach$\mathrm{F}$ the clusters are static once they are designed. In this protocol the cluster head is rotated on a variety of sensor nodes throughout everyone cluster. Leach-F uses the same centralized concept of Leach-C algorithm in order to build the clusters. The method lacks scalability since static clusters do not permit new nodes to be added and the lifespan of such type of network is very less because it never modifies the cluster as nodes dye [41]

\subsubsection{Hybrid Energy Efficient Reactive Protocol} HEER is designed for the reactive protocol. HEER outperforms both DEEC and TEEN in the homogeneous and heterogeneous environment. HEER has more stability period than DEEC as well as TEEN. With HEER the node selected as cluster head is due to their residual energy and moreover. Hard and soft threshold are also introduced in HEER which maximizes the stability period as well as network lifetime. The number of transmissions occur towards base station are limited by hard threshold and number of transmissions occur towards base station are further scrutinized by soft threshold which reduces energy consumption [32].

\subsubsection{DEEC}

DEEC is distributed energy efficient clustering protocol which can be designed for proactive networks. In order to define the cluster head, the node uses the residual as well as the initial energy. This protocol might not need a specific international understanding related energy during each and every selection round. As compare to Leach, the DEEC can perform well in the multi-hop network [19] [21].

\section{RELATED WORK}

Ming Tang et al. [4] proposed Improve Leach protocol to overcome the problem of Leach protocol because leach protocol has some disadvantages like leach protocol does not know about the position of nodes and it also do not have any knowledge related the data that what type of data cluster head sended to base station, also no TDMA is used in this protocol. So to overcome these problems Ming Tang invented an Improved Leach protocol. With the help of this protocol every sensor nodes take out the cluster head transmits detected information in the closest node, not the cluster head.

Mahmoud M.Salim et al. [12] EL-Leach (energy and load balance leach) for get the energy efficiency and load balance. Leach has the drawback that dead nodes are more and it is also not applicable where network deployed in larger regions so in take the more energy. To overcome this drawback improves an EL-Leach. From the result it is prove that this protocol is better than the Leach in parameter better energy efficiency, load balance and network lifetime.

G. Smaragdakis et.al [18] prepared virtually any transmitted, randomized clustering requirements that have got to capable to organize some sort of receptors among the mobile alarm 
system straight into clusters. Chances are they intensive these unique requirements to make some sort of pecking order regarding group leads as well as noticed this particular center gains improved along with the number of periods throughout pecking order. Brings about stochastic geometry were utilized to be able to taken from alternatives for principles of variables of their total criteria this lessened a sum of total energy spent in a network as soon as almost all sensors review information through the entire cluster heads towards the handling center.

Ravi Kishor Izadi et.al [13] In spite of substantial progress within wireless indicator systems (WSNs), vitality resource efficiency remains to be essentially the most significant analysis challenges. Appropriate organization of nodes (clustering) has become the important strategies to grow a life with the entire multilevel via aggregating facts in the chaos head. The actual chaos head will be the spine with the full cluster. This means if the chaos head does not achieve its performance, a obtained plus obtained facts simply by chaos head could be lost. Furthermore, the power utilization pursuing immediate marketing communications through options to help bottom stops will be increased. Izadi avec al. (2013) consist of some sort of type-2 unclear dependent selfconfigurable chaos head assortment $(\mathrm{SCCH})$ method not only to consider the choices requirements with the chaos head but in addition provide a chaos burn approach. Thus, in the event of chaos disappointment, the device however works within an effective way. The actual unique of diet is definitely ale handling connection uncertainty, which in turn is usually an natural functional part of indicator networks. The actual test final results suggest $\mathrm{SCCH}$ functions better than various other not too long ago created methods.

Rohini Sharma et.al [8] projected a distance based cluster head algorithm which might be development of Leach. The aim of wireless sensor network is to achieving communication along with a less amount of power consumptions. To achieve this aim a well-known algorithm, Leach was planned. However leach has some advantages.

Gaurang Rayal et.al [10] provides examining the effectiveness with centralized clustering technique. Within this paper LEACH-Centralized, K Means-CP, practices have been equated regarding clustering along with information supply course of action for various authentic topologies. These simulations had been conducted because of practices as well as enactment on the practices may be seriously examined

Billal.Abu et.al [23] suggested a new mileage dependent cost-effective number of nodes within clustering mechanism. Inside their established program that the LEACH clustering terminates inside of a reduced amount of new release, nevertheless would not give the surety of great chaos mind distribution. Quite a few nodes might decide on a chaos so that the mileage involving $\mathrm{CH}$ to drain (base station) is actually possibly more the distance involving the node per se as well as the drain. In an effort to defend the electrical power of your sensor affiliate networks and increase lifetime, most people advise a new distance-based clustering process, LeachMP (LEACH-minimal path) in order to identify midpoint of the base station to the sensor node, which directly shows the increased network survivability.

Lindsey et.al [6] introduced a Pegasis protocol which is an improvement over Leach. Pegasis is usually a chain based protocol which is an advancement of leach. Unlike Leach, it ignores cluster formation. In this protocol, only one node is chosen a head node which transmits the merged data to the base station for every round. In Pegasis every node communicates through their near neighbor and then takes turn while transmitting to the base station. It works well as compare to LEACH. Pegasis uses a greedy approach and in case if there is any node failure then it bypasses that node. In Pegasis the Data fusion includes a number of data packages from dissimilar sensor dimensions to create a distinct packet. The basic idea of this protocol is to create a chain among the sensor nodes so that everyone node collecting as well as forwarding the data to their near neighbor. [6]

N.M Elashakankiri et.al [41] With report energy-efficient homogeneous clustering protocol that unveiled a whole new means of an energy- useful homogeneous clustering protocol for wi-fi warning cap affiliate networks that the lifespan of your system is increased simply by making certain any homogeneous supply associated with nodes while in the clusters .In the proposed clustering protocol, the bunch users are generally uniformly spread and also the length of the system is additionally extended. Within this report the bunch goes is choosing according to the odds of warning nodes as well as nodes that may have the greater possibility to become a bunch head. In the proposed clustering protocol, the bunch users are generally uniformly spread and also the length of the system is additionally extended

Shio Kumar Singh et.al [7] In paper Quardrature Leach Quadrature Leach increases stability period, network lifetime and throughput moderately considerable.[7] In this, the network can be partitioned into four quadrants to acquire better clustering. TDMA approach is used in Quadratic Leach. Fewer error chances, Fast processing and less time consumption are the advantages of Q-Leach. [7]

Sapna Gambhie et.al [21] a radio warning network (WSN) will be a group of sensors that happen to be geographically distributed plus interlocked by wifi networks. Alarms accumulate info on the condition of bodily world. As a way to give a reverse phone lookup, there are lots of components of conversation strategies that must be explored. Encouraging service quality $(\mathrm{QoS})$ is going to constitute significant importance to get pervasive WSNs this perform the duties of the actual network commercial infrastructure involving diverse applications. To illustrate new information plus development interests in this particular subject, Izadi et aussi al. (2012) reviewed plus talked over the needs, significant challenges, plus open up homework challenges on QoS management with WSNs. A brief overview newest progress will be given.

Mhemed et.al [25] Regardless of sizeable enhancements inside wireless sensor cpa affiliate networks (WSNs), electricity resource efficiency continues to be the most essential exploration challenges. Scientific study has looked into architectures plus topologies which allow power efficient operation connected with WSNs. About the most tactics in this connection is clustering. Even though much scientific study has looked into group scalp collection, Mhemed eaussi al. (2012) investigates the actual group formation.

Salim El Khederi [2] to be able to accumulate data better, cellular warning systems (WSNs) is partitioned directly into clusters. Clustering delivers an easy way to extend your lifetime of WSNs. Present clustering approaches typically employ two approaches: deciding on group leads with more surplus energy, and also revolving group leads frequently, so that you can send out the vitality intake among the nodes around just about every group and also prolong your network lifetime. Having said that, almost all of the earlier algorithms have not considered your expected surplus energy, the predicated left over energy internet marketing determined for a group go and also building a round. Salim .(2012) 
employed, a new fuzzy-logic-based clustering solution by using extra time to your energy predication may be suggested to extend your lifetime of WSNs by way of consistently disseminating your workload.

Kazem Sohraby et.al [35] proposed a data-centric routing to examine that the data sended to base station is relevant or in correct form and reaches to its destination in corrected time or not and they also examine the location of base station.. The data-centric routing shown significant operation acquired over many operational scenarios. Furthermore by evaluated the complexity regarding maximum information aggregation, however it has been NP very difficult trouble in most cases, there exist practical polynomial-time particular cases.

Zhao Han et.al [40] comparison of two routing protocol Leach and Pegasis. The evolution is done on basis of different argument like a total energy consumed, overheads and sensors lifetime. In Leach, limited data processing occurs at specified nodes called cluster-heads and lastly collected data is sended to the sink node. While in Pegasis, there is no data aggregation occurs. Sensoria simulator is used for simulation.

\section{COMPARATIVE ANALYSIS OF VARIOUS HIERARCHICAL ROUTING PROTOCOLS}

There are various popular hierarchical protocol that have been compared by using various parameters which shows that each protocols has their own stability for choosing cluster head. The comparisons of hierarchical protocol are described in Table1.

Table1. Comparison of hierarchical routing protocols

\begin{tabular}{|c|c|c|c|c|c|}
\hline PROTOCOL & MOBILITY & HOP COUNT & $\begin{array}{c}\text { ENERGY } \\
\text { EFFICIENCY }\end{array}$ & SCALABILITY & ROUTING \\
\hline Leach & Fixed & Single hop & Low & Good & Cluster Based \\
\hline Pegasis & Fixed & Multi hop & Low & Good & Chain Based \\
\hline Leach- C & Fixed & Single hop & Very High & Good & Cluster Based \\
\hline Leach-F & Fixed & Single hop & High & Limited & Cluster Based \\
\hline TEEN & Fixed & Single hop & Very High & Good & Hybrid \\
\hline APTEEN & Fixed & Single hop & Moderate & Good & Hybrid \\
\hline $\mathrm{HEEb}$ & Stationary & Multi hop & Moderate & Good & Cluster Based \\
\hline EARP & Stationary & Multi hop & High & High & Cluster Based \\
\hline SA-Leach & Variable & Single hop & High & Good & Cluster Based \\
\hline O-Leach & Variable & Single hop & High & Good & Cluster Based \\
\hline EEZECR & Variable & Multi hop & High & Good & Cluster Based \\
\hline $\mathrm{ELCH}$ & Fixed & Single hop & High & Limited & Cluster Based \\
\hline HPAR & No & Single hop & High & Low & Cluster Based \\
\hline SHPER & Fixed & Single hop & High & Good & Cluster Based \\
\hline DHAC & No & Multi hop & Low & Good & Cluster Based \\
\hline EEHC & Fixed & Single Hop & Low & Very Low & Cluster Based \\
\hline DDAR & No & Multi hop & Medium & Medium & Cluster Based \\
\hline O-Leach & Variable & Single hop & Very High & Very good & Cluster Based \\
\hline EELP & Fixed & Single hop & Very High & Good & Cluster Based \\
\hline Cog-Leach & Variable & Single hop & Very High & Good & Cluster Based \\
\hline Leach-SM & Variable & Single hop & Very High & Good & Cluster Based \\
\hline AQM-Leach & Variable & Single hop & High & Limited & Cluster Based \\
\hline I-Leach & Variable & Multi hop & Very High & Good & Cluster Based \\
\hline E-Leach & Variable & Single hop & Moderate & Good & Cluster Based \\
\hline Multi-level Leach & Variable & Multi hop & High & Medium & Cluster Based \\
\hline Op-Leach & Variable & Single hop & Moderate & Medium & Cluster Based \\
\hline $\begin{array}{c}\text { Leach with Mobile } \\
\text { Sink }\end{array}$ & Variable & Multi hop & High & Medium & Cluster Based \\
\hline
\end{tabular}




\begin{tabular}{|c|c|c|c|c|c|}
\hline PR-Leach & Variable & Single hop & Moderate & Good & Cluster Based \\
\hline Q-Leach & Variable & Multi hop & Very High & Good & Cluster Based \\
\hline $\mathrm{DBCH}$ & Fixed & Multi hop & Very High & Good & Cluster Based \\
\hline DWEHC & Fixed & Multi hop & Moderate & Medium & Cluster Based \\
\hline PANEL & Fixed & Single hop & Moderate & Medium & Cluster Based \\
\hline A-Leach & Fixed & Single hop & Very High & Good & Cluster Based \\
\hline Leach-A & Fixed & Single hop & Very High & Good & Cluster Based \\
\hline Leach-B & Fixed & Single hop & Very High & Good & Cluster Based \\
\hline Leach-C & Fixed & Single hop & Very High & Very Good & Cluster Based \\
\hline Leach-E & Variable & Single hop & Very High & Very Good & Cluster Based \\
\hline Leach-F & Variable & Single hop & Very High & Limited & Cluster Based \\
\hline Leach-M & $\begin{array}{c}\text { Mobile BS and } \\
\text { Nodes }\end{array}$ & Multi hop & Very High & Very Good & Cluster Based \\
\hline Leach-L & Fixed & Multi hop & Very High & Good & Cluster Based \\
\hline Leach-S & Fixed & Single hop & Very High & Very Good & Cluster Based \\
\hline V-Leach & Fixed & Single hop & Very High & Very Good & Cluster Based \\
\hline TL-Leach & Fixed & Single hop & Very High & Very Good & Cluster Based \\
\hline Leach-E & Fixed & Single hop & High & Good & Cluster Based \\
\hline RLeach & Fixed & Multi hop & Good & Good & Cluster Based \\
\hline
\end{tabular}

\section{CONCLUSION AND FUTURE RESEARCH}

Modern advancement in wireless communications have permitted the creation of low-cost, low-power WSNs along with large applicability. The main aim of hierarchical routing protocols is to increase the lifespan of network, reducing power depletion of finest sensor networking protocols and algorithms. In this paper, we have surveyed as well as summarized current research works focused mainly on the energy efficient hierarchical cluster based routing protocols for WSNs. In this paper we have described only a few section of routing protocols. The protocols deliberated in this paper have discrete advantages and disadvantages. Based on the topology, the protocol and routing strategies are applied according to its topology. We have also developed a novel taxonomy of clustering routing methods for WSNs based on rather detailed clustering attributes. Finally we have described a comparison of routing protocols according to its taxonomy and parameters. The factors which are affected cluster formation and $\mathrm{CH}$ communication are key issue for future research.

\section{REFERENCES}

[1] W. Heinzelman, A. Chandrakasan and H. Balakrishnan. 2000. Energy-Efficient Communication Protocol for Wireless Microsensor Networks. Proceedings of the 33rd Hawaii International Conference on System Sciences (HICSS '00).

[2] Salim El Khediri, Nejah Nasri, Anne Wei, Abdennaceur Kachouri, A New Approach for Clustering in Wireless Sensors Networks Based on LEACH, Procedia Computer Science, Vol. 32,pp. 1180-1185.
[3] Abdullah Erdal Tumer, Mesut Gunduz, An improved leach protocol for indoor wireless sensor networks, in Proceedings of IEEE International Conference on Signal Processing and Integrated Networks (SPIN'14), Noida, 20-21 February 2014, pp. 432-437.

[4] Yanhong Sun, Ming Tang, An Enhanced Protocol for LEACH Based Wireless Sensor Networks, in Proceedings of the IEEE International Symposium on Computer, Consumer and Control (IS3C'14), Taichung, 10-12 June 2014, pp. 344-347.

[5] Feng Zhao, Leonidas Guibas, Wireless Sensor Networks - An Information Processing Approach, Morgan Kaufmann Publishers, 2004.

[6] S. Lindsey, C. Raghavendra. 2002. PEGASIS: Power Efficient Gathering in Sensor Information Systems. IEEE Aerospace Conference Proceedings, Vol. 3, 9-16 pp. 11251130 .

[7] Shio Kumar Singh et.al Energy efficient Homogeneous Clustering Algorithm for WSN, journal 2010 B.Manzoor,N.Javid,O.rehman,M.Akbar,Q.Nadeem” QLeach: A new Routing Protocol for WSNs" Elsevier 2013

[8] Rohini Sharma, Narendra Mishra, Dr Sumit Srivastva “ A proposed energy efficient distance based cluster head algorithm: An improvement over leach" Elsevier 2015

[9] Jenn-Long Liu and Chinya V. Ravishankar, Member “ LEACH-GA: Genetic Algorithm-Based Energy-Efficient Adaptive Clustering Protocol for Wireless Sensor Networks " IEEE 2011 
[10] Gaurang Raval , Analyzing the Performance of Centralized Clustering Techniques for Realistic Wireless Sensor Network Topologies, Elsevier,2015

[11] Hari Prabhat "Geographic Routing In Clustered Wireless Sensor Network Among Obstacles" IEEE,2015BASE PAPER

[12] Mahmoud M. Salim, Hussein A. Elsayed, Salwa H. El Ramly, PR-LEACH: Approach for Balancing Energy Dissipation of LEACH Protocol for Wireless Sensor Networks, in Proceedings of the IEEE 31st National Radio Science Conference (NRSC'14), Cairo, 28-30 April 2014, pp. 252-259.

[13] Ravi Kishore Kodali, Naveen Kumar Aravapalli, Multilevel LEACH Protocol model using NS-3, in Proceedings of the IEEE International Advance Computing Conference (IACC'14), Gurgaon, 21-22 February 2014, pp. 375-380.

[14] A. Manjeshwar and D. P. Agarwal. 2001. TEEN: a routing protocol for enhanced efficiency in wireless sensor networks. In 1st International Workshop on Parallel and Distributed Computing Issues in Wireless Networks and Mobile Computing.

[15] A. Manjeshwar and D. P. Agarwal. 2001. TEEN: a routing protocol for enhanced efficiency in wireless sensor networks. In 1st International Workshop on Parallel and Distributed Computing Issues in Wireless Networks and Mobile Computing

[16] A. Manjeshwar and D. P. Agarwal. 2002. APTEEN: A hybrid protocol for efficient routing and comprehensive information retrieval in wireless sensor networks. Parallel and Distributed Processing Symposium, Proceedings International, IPDPS, pp. 195-202

[17] Q. Li, Z. Qingxin, and W. Mingwen. 2006. Design of a distributed energy efficient clustering algorithm for heterogeneous wireless sensor networks. Computer Communications, vol. 29, pp. 2230-7

[18] G. Smaragdakis, I. Matta, A. Bestavros. 2004. SEP: A Stable Election Protocol for clustered heterogeneous wireless sensor networks. In Second International Workshop on Sensor and Actor Network Protocols and Applications (SANPA).

[19] Ossama Younis and Sonia Fahmy. 2004. Distributed Clustering in Ad-hoc Sensor Networks: A Hybrid, EnergyEfficient Approach. In Proceedings of IEEE INFOCOM, Hong Kong, an extended version appeared in IEEE Transactions on Mobile Computing, 3(4)

[20] Younis, O.; Fahmy,S HEED: A hybrid, energy-efficient, distributed clustering approach for adhoc sensor networks.

[21] IEEETrans.Mobile Comput. 2004, 3, 366-379. RidhaSouaand Pascale Minet, " A Survey on Energy Efficient Techniques in Wireless Sensor Networks,'IEEE Technical Program at IFIP WMNC' 2011.

[22] Sapna Gambhir, Nida Fatima, Op-LEACH: An Optimized LEACH Method for busty traffic in WSNs, in Proceedings of the IEEE Fourth International Conference on Advanced Computing \& Communication Technologies (ACCT'14), Rohtak, 8-9 February 2014, pp. 222-229.
[23] Mohammad Shurman, Noor Awad, Mamoun F. AlMistarihi, Khalid A. Darabkh, LEACH Enhancements for Wireless Sensor Networks Based on Energy Model, in Proceedings of the IEEE 11thInternational MultiConference on Systems, Signals\& Devices (SSD'14), Barcelona, 11-14 February 2014, pp. 1-4.

[24] Bilal Abu Bakr, Leszek T. Lilien, Comparison by Simulation of Energy Consumption and WSNLifetime for LEACH and LEACH-SM, Procedia Computer Science, Vol. 34, 2014, pp. 180-187.

[25] Joseph Polastre, Robert Szewczyk, Alan Mainwaring, David Culler, John Anderson, Analysisof Wireless Sensor Networks for Habitat Monitoring, Wireless sensor Networks, 2004, pp. 399-423.

[26] Mahmoud M. Salim, Hussein A. Elsayed, Salwa H.El Ramly, PR-LEACH: Approach for Balancing Energy Dissipation of LEACH Protocol for Wireless Sensor Networks, in Proceedings of the IEEE 31st National Radio Science Conference(NRSC'14), Cairo, 28-30 April 2014, pp. 252-259.

[27] Rashad M. Eletreby, Hany M. Elsayed, Mohamed M. Khairy, CogLEACH: A Spectrum Aware Clustering Protocol for Cognitive Radio Sensor Networks, in Proceedings of the 9th International Conference on Cognitive Radio Oriented Wireless Networks and Communications (CROWNCOM'14), Oulu, 2-4 June2014, pp. 179-184.

[28] Manjeshwar, E.; Agrawal, D.P. TEEN: A Routing Protocol for Enhanced Efficiency in Wireless Sensor Networks In Proceedings of the 15th International Parallel and Distributed Processing Symposium (IPDPS), San Francisco, CA,USA, 23-27 April 2001; pp. 20092015.

[29] Muhammad Akhlaq, Member, IEEE, and Tarek R. Sheltami, Member, IEEE RTSP: An Accurate and Energy-Efficient Protocol for Clock Synchronization in WSNs IEEE TRANSACTIONS ON INSTRUMENTATION AND MEASUREMENT, VOL. 62, NO. 3, MARCH 2013.

[30] Rashmi Ranjan Rout, Student Member, IEEE, and Soumya K. Ghosh, Member, IEEE Enhancement of Lifetime using Duty Cycle and Network Coding in Wireless Sensor Networks IEEE TRANSACTIONS ON WIRELESS COMMUNICATIONS, VOL. 12, NO. 2, FEBRUARY 2013.

[31] Zhao Han, Jie Wu, Member, IEEE, Jie Zhang, Liefeng Liu, and Kaiyun Tian A General Self-Organized TreeBased EnergyBalance Routing Protocol for Wireless Sensor Network IEEE TRANSACTIONS ON NUCLEAR SCIENCE, VOL. 61, NO.2, APRIL 2014.

[32] K. Padmanabhan, Dr. P. Kamalakkannan "Energy Efficient Adaptive Protocol for Clustered Wireless SensorNetworks" IJCSI International Journal.

[33] Harneet Kour and Ajay K. Sharma, "Hybrid Energy Efficient Distributed Protocol for Heterogeneous Wireless Sensor Network", International Journal of Computer Applications (0975 - 8887) Volume 4 - No.6, July 2010.

[34] I.F. Akyildiz, W. Su*, Y. Sankarasubramaniam, E. Cayirci, "Wireless sensor networks: a Survey", Broadband and Wireless Networking 
[35] P. Minet, "Energy Efficient Routing", in Ad Hoc and Sensor Wireless Networks: Architectures: Algorithms and Protocols. Bentham Science 2009.

[36] Kazem Sohraby, Daniel Minoli, Taieb F. Znati, "Wireless sensor networks:technology,protocols and applications",A John Wiley \& Sons ,Inc.,Publication 2007

[37] I. F. Akyildiz, W. Su, Y. Sankarasubramaniam and E. Cayirci, "Wireless Sensor Networks: A survey," Computer Networks, Volume 38, N. 4, March 2011.

[38] Moslem Afrashteh Mehr, "Design and Implementation a New Energy Efficient Clustering Algorithm using Genetic Algorithm for Wireless Sensor Networks" World Academy of Science, Engineering and Technology, 52 ,2011Parminder Kaur, Mrs. Mamta Katiyar, "The Energy-Efficient Hierarchical Routing Protocols for WSN: A Review"(International Journal of advanced research in computer science), volume 2,issue 11,2012.

[39] Younis, O.; Fahmy,S HEED: A hybrid, energy-efficient, distributed clustering approach for adhoc sensor networks. IEEE Trans. Mobile Comput. 2004, 3, 366-
379. RidhaSouaand Pascale Minet, "A Survey on Energy Efficient Techniques in Wireless Sensor Networks,'IEEE Technical Program at IFIP WMNC' 2011.

[40] T. N.Qureshi, T. Shah and N. Javaid. EESAA: Energy Efficient Sleep Awake Aware Intelligent Sensor Network Routing Protocol. In Proceedings of 15th International Multi Topic Conference (INMIC), Islamabad, PK (13th to 15 th December 2012).

[41] Zhao Han, Jie Wu, Member, IEEE, Jie Zhang, Liefeng Liu, and Kaiyun Tian A General Self-Organized TreeBased EnergyBalance Routing Protocol for Wireless Sensor Network IEEE TRANSACTIONS ON NUCLEAR SCIENCE, VOL. 61, NO.2, APRIL 2014.

[42] N.M. Elshakankiri, N. M. Moustafa and Y. H. Dakroury, -Energy Efficient Routing Protocol for Wireless Sensor Network\| in IEEE InternationalConference on pp. 393 398,December 2008

[43] J. Al-Karaki, and A. Kamal, .Routing Techniques in Wireless Sensor Networks: A Survey., IEEE Communications Magazine, vol 11, no. 6, Dec. 2004,pp. 6-28. 